\title{
THE GRAVITATIONAL LENS-GALAXY GROUP CONNECTION. II. GROUPS ASSOCIATED WITH B2319+051 AND B1600+434
}

\author{
M. W. Auger, C. D. Fassnacht, A. L. Abrahamse, and L. M. Lubin \\ Department of Physics, University of California, 1 Shields Avenue, Davis, CA 95616, USA; \\ mauger@physics.ucdavis.edu, fassnacht@physics.ucdavis.edu \\ AND \\ G. K. SQUiRES \\ Spitzer Science Center, California Institute of Technology, Mail Code 220-6, \\ 1200 East California Boulevard, Pasadena, CA 91125, USA \\ Received 2006 March 16; accepted 2007 April 27
}

\begin{abstract}
We report on the results of a spectroscopic survey of the environments of the gravitational lens systems CLASS $\mathrm{B} 1600+434\left(z_{l}=0.41, z_{s}=1.59\right)$ and CLASS B2319+051 $\left(z_{l}=0.62\right)$. The B1600+434 system has a time delay measured for it, and we find that the system lies in a group with a velocity dispersion of $90 \mathrm{~km} \mathrm{~s}^{-1}$ and at least seven members. B2319+051 has a large group in its immediate foreground with at least 10 members and a velocity dispersion of $460 \mathrm{~km} \mathrm{~s}^{-1}$ and another in the background of the lens with a velocity dispersion of $190 \mathrm{~km} \mathrm{~s}^{-1}$. These systems increase the sample of spectroscopically confirmed groups associated with strong lenses by $20 \%$. We find that modeling groups as single group-sized halos produces only small corrections $\left(\kappa_{\text {env,grp }} \sim 0.01\right)$ to lensing models. However, the very local environments, i.e., galaxies within $\sim 15^{\prime \prime}$ of the lensing galaxy, have stronger effects on the lens models of the systems studied here. We quantify the effects of the local environment on the lens models and find up to an $\approx 10 \%$ correction to the derived value of $H_{0}$ for B1600+434 compared to models that do not account for the lens environment.
\end{abstract}

Key words: distance scale — galaxies: clusters: general — galaxies: individual (B2319+051, B1600+434) — gravitational lensing

\section{INTRODUCTION}

Galaxy groups are a fundamental component of the largescale structure of the universe, and over half of all galaxies in the local universe are members of groups (e.g., Tully 1987; Ramella et al. 1989). Precision cosmology relies on an accurate knowledge of the distribution of galaxies in order to model the galaxy power spectrum properly (e.g., Evrard et al. 2002; Yang et al. 2004), and it is essential that galaxy groups are identified to describe the small-scale, nonlinear regime of the matter power spectrum accurately. Several local redshift surveys, including the Sloan Digital Sky Survey (SDSS; York et al. 2000) and the Two Degree Field Galaxy Redshift Survey (2dFGRS; Colless et al. 2001), have produced results using groups out to $z \sim 0.2$ (Weinmann et al. 2006; Collister \& Lahav 2005; Abazajian et al. 2005; Padilla et al. 2004). At higher redshifts, the Canadian Network for Observational Cosmology redshift survey (CNOC2; Carlberg et al. 2001a) probes redshifts in the range $0.15 \lesssim z \lesssim 0.5$, while the DEEP2 Galaxy Redshift Survey is probing redshifts in the range $0.7 \lesssim z \lesssim 1$ (Coil et al. 2004, 2006). Weak lensing analyses of groups also promise to yield information about the mass distribution in galaxy groups (e.g., Möller et al. 2002; Faure et al. 2004; Hoekstra et al. 2001). Knowledge of group substructure can then be used to confirm or refute dark matter models (Reed et al. 2005; D'Onghia \& Lake 2004).

Groups also hold significance for strong gravitational lensing. Keeton et al. (2000) estimate that $25 \%$ of lenses lie in group environments, although the fraction could be much higher (e.g., Williams et al. 2006; Blandford et al. 2001; Oguri et al. 2005). A moderately massive group that is sufficiently close to the lensing galaxy can contribute to the convergence in the lensing potential and alter the shear field of the lens. Theoretical estimates of the importance of these contributions have been made (e.g., Oguri et al. 2005; Keeton \& Zabludoff 2004; Möller et al. 2002), but it remains unclear to what extent lens models must account for group environments (e.g., Momcheva et al. 2006; Fassnacht et al. 2006; Morgan et al. 2005; Dalal \& Watson 2004). Furthermore, gravitational lens time-delay measurements and improved mass-modeling techniques have lowered the uncertainties in measurements of the Hubble constant, $H_{0}$, to the $\approx 10 \%$ level (e.g., Koopmans et al. 2003; Kochanek \& Schechter 2004), and the systematic errors introduced by ignoring lens environments now contribute significantly to the $H_{0}$ error budget.

In addition to improving the determination of cosmological parameters, galaxy groups also allow us to study the evolution of galaxies. Group environments are likely locations for mergers (e.g., Zabludoff \& Mulchaey 1998; Carlberg et al. 2001b; Aarseth \& Fall 1980) compared to clusters or the field (e.g., Aceves \& Velázquez 2002; Lin et al. 2004; Conselice et al. 2003; Patton et al. 2002). Mergers and nonmerger interactions in groups can drive changes in galaxy morphologies and star formation rates (SFRs) that are suppressed in more dense environments (e.g., Zabludoff \& Mulchaey 1998; Bower \& Balogh 2004). Furthermore, active galactic nuclei (AGNs) are expected to reside in dense environments where galaxy-galaxy interactions provide mechanisms for fueling AGNs (e.g., Best 2004; Bahcall et al. 1997), although the validity of this claim is uncertain (e.g., Kauffmann et al. 2004; Miller et al. 2003). Best et al. (2005) only find a correlation between radio-loud AGNs and the local environment, while McLure \& Dunlop (2001) find no distinction between radio-loud and radio-quiet AGN populations in clusters. 
TABLE 1

Lens System Imaging

\begin{tabular}{ccccc}
\hline \hline \multirow{2}{*}{ Lens System } & Band & $\begin{array}{c}\text { Exposure Time } \\
(\mathrm{s})\end{array}$ & Instrument & Date \\
\hline B2319+051................... & $R$ & 1200 & LRIS & 1998 Aug 1 \\
& $B$ & 900 & LRIS & 1999 Aug 16 \\
& $V$ & 3900 & LRIS & 1999 Aug 16 \\
& $I$ & 2700 & LRIS & 1999 Aug 16 \\
& F555W & 4800 & WFPC2 & 2000 Sep 27 \\
& F814W & 4800 & WFPC2 & 2000 Sep 27 \\
& $B$ & 1200 & Suprime-Cam & 2001 May 21 \\
& $R$ & 2700 & Suprime-Cam & 2001 Apr 25 \& 26 \\
& $I$ & 1380 & Suprime-Cam & 2001 Apr 20 \\
& F555W & 5300 & WFPC2 & 2001 Jun 16 \\
& F555W & 18200 & WFPC2 & 2001 Sep 21-23 \\
& F814W & 7800 & WFPC2 & 2001 Sep 26 \\
\hline
\end{tabular}

We are currently conducting a systematic survey to investigate the environments of strong gravitational lens systems (e.g., Fassnacht \& Lubin 2002; Fassnacht et al. 2006). The final sample of groups from our study will be compared with groups observed in the local universe (Merchán \& Zandivarez 2005; Balogh et al. 2004) and with recent observations of other moderate-redshift groups (Wilman et al. 2005a, 2005b; Momcheva et al. 2006; Williams et al. 2006; Gerke et al. 2005; Jeltema et al. 2006) to quantify the evolution of groups with redshift. In this paper we present the discovery of three groups associated with the lens systems B1600+ 434 (Jackson et al. 1995) and B2319+051 (Rusin et al. 2001), hereafter B1600 and B2319, respectively. These lenses were discovered by the Cosmic Lens All-Sky Survey (CLASS; Myers et al. 2003; Browne et al. 2003). In the case of B2319, the lens is not found to be part of a group, but there is a large group in the immediate foreground of the lens and another group in the background of the lens. In contrast, B1600 is found to be a member of a poor group of galaxies. These observations bring the total number of spectroscopically confirmed lens-group associations to seven: B1600, B1608+656, B1422+231, PG 1115+080, MG 0751+2716, MG 1654+1346, and B2114+022 (Fassnacht et al. 2006; Kundic et al. 1997a, 1997b; Tonry 1998; Tonry \& Kochanek 1999; Momcheva et al. 2006). In addition, there are now four strong lens systems with spectroscopically confirmed groups in the immediate foreground and/or background of the lenses: B2319, B0712+472, HE 0435-1223, and MG 1131+0445 (Fassnacht \& Lubin 2002; Morgan et al. 2005; Tonry \& Kochanek 2000). The large number of interloper systems may be an indication that lenses lie along preferentially overdense lines of sight (e.g., C. D. Fassnacht et al. 2007, in preparation). Furthermore, Momcheva et al. (2006) find a group at the expected redshift of BRI 09520115, although no spectroscopic redshift has been obtained for the lens to confirm the association. Here we examine the effects of these new groups on the lens models for each system, quantifying the contributions of the environments to the shears and convergences of the lens systems.

There are approximately 150 published strong gravitational lens systems, and many of these require small ad hoc additional shear and convergence components to be adequately modeled (e.g., Lehár et al. 2000). The B2319 system $\left(z_{l}=0.62\right)$ requires a large shear component $(\gamma=0.14)$ to fit acceptable models, and this shear cannot be entirely accounted for by the lensing galaxy (Rusin et al. 2001). There is a secondary lensing candidate $3.4^{\prime \prime}$ away from the primary lens, and the redshifts of the lensing and secondary galaxies are 0.624 and 0.588 , respectively (Lubin et al. 2000). How- ever, it is likely that the secondary galaxy alone cannot account for the shear discrepancy (assuming a velocity dispersion of $180 \mathrm{~km} \mathrm{~s}^{-1}$ for the neighboring galaxy and a singular isothermal sphere [SIS] profile, the shear contribution from this second galaxy would be $\gamma \approx 0.07$ ), and a more massive, grouplike structure may be required to reproduce the observed image configuration. The $\mathrm{B} 1600$ system $\left(z_{l}=0.41, z_{s}=1.59\right)$ has a reasonably well-constrained lens model (Koopmans et al. 1998; Maller et al. 2000), but initial spectroscopy of the environment of the lensing galaxy has revealed several nearby galaxies with redshifts similar to the lensing galaxy. It is crucial that the group associated with B1600 be investigated due to the importance of this system in determining $H_{0}$ (Koopmans et al. 2000; Maller et al. 2000; Kochanek 2002, 2003; Burud et al. 2000).

\section{OBSERVATIONS AND DATA REDUCTION}

\subsection{Imaging}

We have obtained deep nonphotometric BVRI images of B2319 using the Low-Resolution Imaging Spectrometer (LRIS; Oke et al. 1995) and Echellette Spectrograph and Imager (ESI; Sheinis et al. 2002) instruments on the Keck telescopes. We have also obtained photometric LRIS snapshots of the field in the $B V I$ filters, allowing us to photometrically calibrate our deeper imaging in these bands. These Keck imaging data were reduced using standard IRAF ${ }^{1}$ tasks. Our primary imaging of B1600 comes from deep BRI imaging from the Suprime-Cam instrument (Miyazaki et al. 2002) on the Subaru Telescope, obtained from the SMOKA archive (Baba et al. 2002). These data were reduced using the SDFRED package (Ouchi et al. 2004). A comparison between stars in our Suprime-Cam imaging and SDSS imaging of the field of B1600 (after applying Lupton's transformation ${ }^{2}$ between the two different filter sets) allowed us to establish a photometric zero point for the Suprime-Cam archival data. In addition, we have Hubble Space Telescope (HST) imaging of both fields with the WFPC2 camera in the F555W and F814W filters. A summary of our imaging data can be found in Table 1.

\footnotetext{
${ }^{1}$ IRAF is distributed by the National Optical Astronomy Observatory, which is operated by the Association of Universities for Research in Astronomy, Inc., under cooperative agreement with the National Science Foundation.

${ }^{2}$ We used the filter transformations that had smaller reported values for $\sigma$. Lupton's filter transformation equations can be found on the SDSS Data Release 4 Web site at http://www.sdss.org/dr4/algorithms/sdssUBVRITransform.html.
} 
TABLE 2

Lens Field LRIS Spectroscopy

\begin{tabular}{cccc}
\hline \hline Lens System & $\begin{array}{c}\text { Number of } \\
\text { Redshifts }\end{array}$ & $\begin{array}{c}\text { Exposure Time } \\
(\mathrm{s})\end{array}$ & Date \\
\hline B2319+051....... & 8 & 5400 & 2001 Jul 26 \\
& 21 & 8100 & 2002 Jul 15 \\
& 12 & 3600 & 2002 Jul 16 \\
B1600+434....... & 15 & 5400 & 2003 Aug 1 \\
& 21 & 5400 & 2003 Jul 31 \\
& 19 & 3600 & 2006 Jun 20 \\
\hline
\end{tabular}

\subsection{Spectroscopy}

A color selection was applied to choose candidate group members for the two lens systems. These colors were chosen based on the colors of the lensing galaxy or colors of nearby galaxies known to be at approximately the same redshift as the lens from initial long-slit spectroscopy of the systems. Target galaxies generally had colors within $0.1 \mathrm{mag}$ of the color criteria and were within $\sim 3^{\prime}$ of the lensing galaxy. This corresponds to approximately 3 times the virial radii of typical groups, or $775 h^{-1} \mathrm{kpc}$ at the redshift of B1600 and $970 h^{-1} \mathrm{kpc}$ at the redshift of B2319 for a $\Lambda \mathrm{CDM}$ cosmology. Our spectral candidates were selected to have $R \lesssim 22$, although some fainter candidates were selected to fill open spaces on the slit masks. Four slit masks were taken for the field of B2319, and two slit masks were obtained for the field of B1600 with LRIS (Table 2). A handful of additional redshifts were also obtained from long-slit spectra of each lens system with LRIS and ESI. The spectra were flat-fielded using internal flat lamps, and wavelength solutions were derived using internal spectral lamps. Most of the spectra were reduced using standard IRAF tasks, although the second B1600 mask was reduced using a custom LRIS reduction pipeline. Redshifts for each spectrum were determined by finding at least one emission line and one other feature, or by identifying multiple absorption features in each spectrum. Identified spectral features typically had signal-to-noise ratios of at least $\mathrm{S} / \mathrm{N}=5$, and redshift errors were determined by finding the rms deviation of the centroids of these features with respect to their expected centroids; these errors are typically $\Delta z \approx 0.0004$. In total, we have 53 redshifts for the B2319 field and 44 redshifts for the B1600 field. (The number of redshifts in Table 2 does not reflect our long-slit spectra but does include multiple observations of some target galaxies; all targets with repeated observations have redshifts that agree within the measurement errors.)

\section{GROUP IDENTIFICATION}

The redshift distributions obtained from the spectroscopy of our two target lens systems are shown in Figures 1 and 2. There are two obvious peaks in the field of B2319 and one clear peak at the lens redshift of B1600. We identify possible groups by initially associating all galaxies that are within $\delta z=0.005$ of each other (in effect, we take each spike from the redshift distribution to be a potential group). We then use the formalism of Wilman et al. (2005a) to exclude nonmembers and to determine the group's velocity dispersion. That is, we find the average redshift and position of the potential group members (clipping the extreme members for groups with more than three potential members), define a first-approximation observed velocity dispersion of $\sigma_{\text {obs }}=$ $350(1+\bar{z}) \mathrm{km} \mathrm{s}^{-1}$, set an initial redshift shell

$$
\delta z=\frac{2 \sigma_{\mathrm{obs}}}{c},
$$

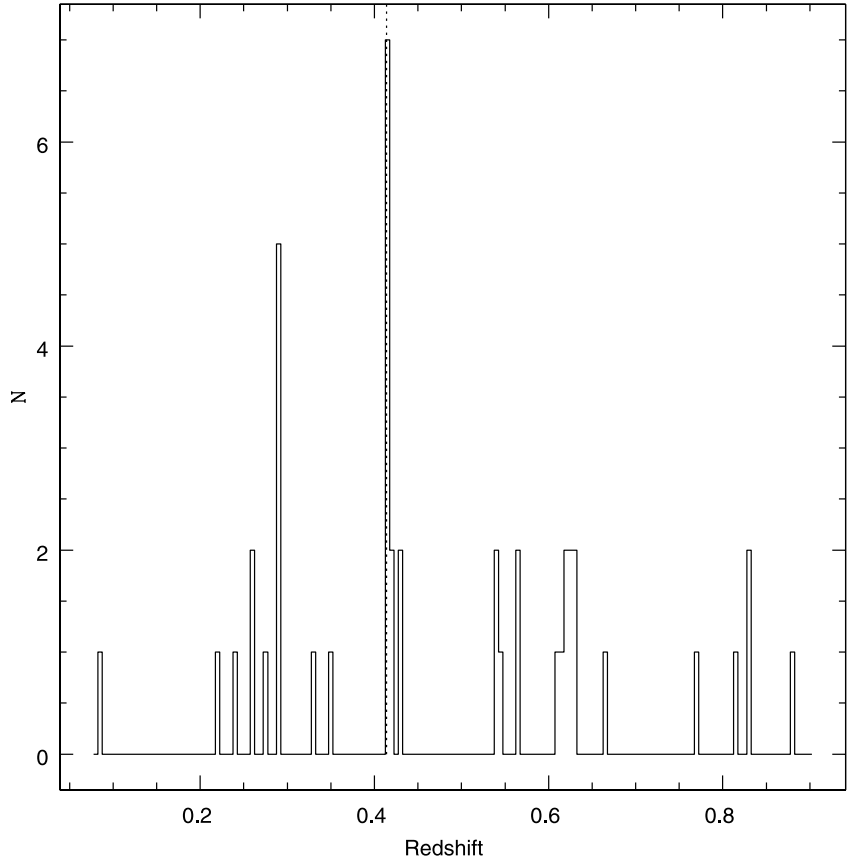

FIg. 1.-Redshift distribution of galaxies in the field of the gravitational lens B1600. The redshift of the lens (dotted line) is 0.41 .

and specify a maximum angular radius based on this redshift shell,

$$
\delta \theta=206,265^{\prime \prime} \frac{c \delta z}{b(1+\bar{z}) H_{0} D_{\theta}} .
$$

Here $D_{\theta}$ is the angular diameter distance to the mean redshift. We assume a $\Lambda$ CDM cosmology with $\Omega_{m}=0.27$ and $\Omega_{\Lambda}=0.73$, and we follow Wilman et al. (2005a) in fixing our aspect ratio, $b=3.5$. Note that the initial approximation of $\sigma=350 \mathrm{~km} \mathrm{~s}^{-1}$

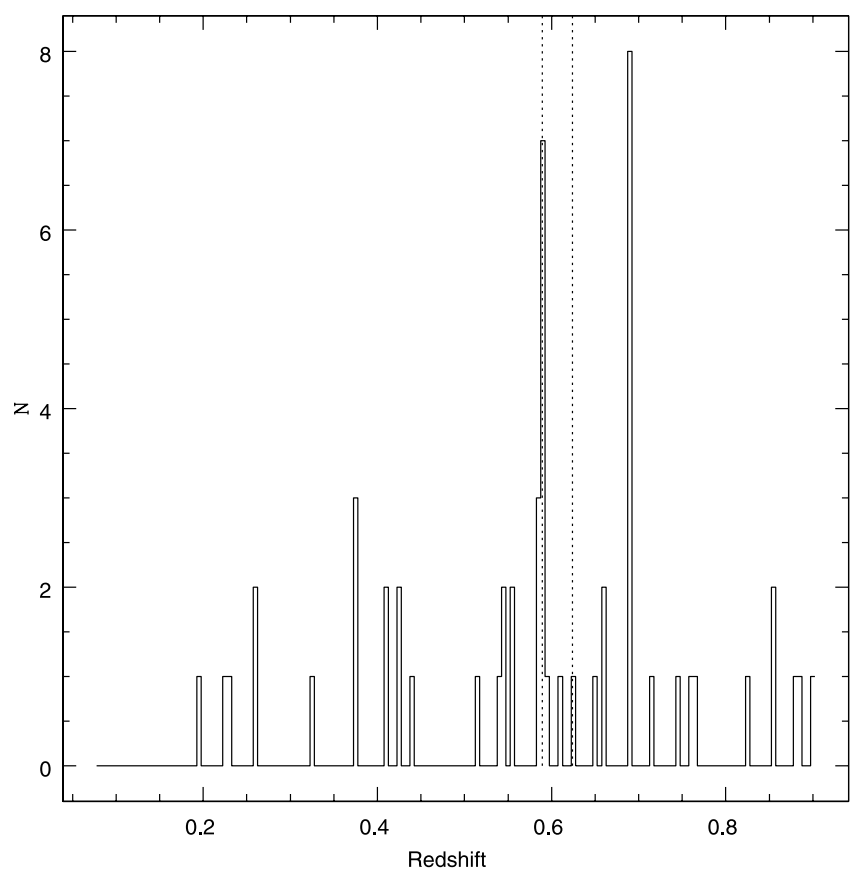

FIG. 2.- Redshift distribution of field galaxies for the gravitational lens B2319. The redshift of the lens (dotted line) is 0.62 ; the redshift of the secondary lensing galaxy is also marked, at $z=0.59$. 
TABLE 3

Summary of Galaxy Groups in the Fields of B1600 and B2319

\begin{tabular}{|c|c|c|c|c|c|c|c|c|c|}
\hline Field & R.A. & Decl. & $z$ & $N_{\text {mem }}$ & $\begin{array}{c}\sigma \\
\left(\mathrm{km} \mathrm{s}^{-1}\right)\end{array}$ & $\begin{array}{c}M_{\mathrm{vir}} \\
\left(10^{14} h M_{\odot}\right)\end{array}$ & $\begin{array}{c}R_{\mathrm{vir}} \\
\left(h^{-1} \mathrm{Mpc}\right)\end{array}$ & $\begin{array}{c}R_{p} \\
\left(h^{-1} \mathrm{Mpc}\right)\end{array}$ & $\begin{array}{c}t_{c} \\
\text { (Hubble Times) }\end{array}$ \\
\hline \multirow[t]{4}{*}{ B1600 ...................... } & 160140 & 431648 & 0.415 & 7 & $90 \pm 30$ & 0.02 & 0.17 & 0.31 & 0.05 \\
\hline & 160140 & 431800 & 0.540 & 3 & $640 \pm 770$ & 5.6 & 1.01 & 0.93 & 0.04 \\
\hline & 160139 & 431511 & 0.623 & 4 & $590 \pm 450$ & 2.9 & 0.61 & 0.94 & 0.03 \\
\hline & 160149 & 431724 & 0.291 & 5 & $337 \pm 120$ & 0.69 & 0.45 & 0.64 & 0.04 \\
\hline \multirow[t]{4}{*}{ B2319.................... } & 232139 & 052652 & 0.589 & 10 & $460 \pm 80$ & 0.67 & 0.24 & 0.42 & 0.01 \\
\hline & 232140 & 052648 & 0.689 & 8 & $190 \pm 50$ & 0.15 & 0.32 & 0.41 & 0.05 \\
\hline & 232141 & 052743 & 0.375 & 3 & $340 \pm 240$ & 0.64 & 0.43 & 0.62 & 0.03 \\
\hline & 232140 & 052621 & 0.542 & 3 & $260 \pm 290$ & 1.03 & 1.10 & 1.16 & 0.11 \\
\hline
\end{tabular}

NoтE.-Units of right ascension are hours, minutes, and seconds, and units of declination are degrees, arcminutes, and arcseconds.

makes the algorithm relatively insensitive to high velocity dispersion structures $\left(\sigma \gtrsim 1000 \mathrm{~km} \mathrm{~s}^{-1}\right)$; the lack of a substantial overdensity of red galaxies indicates that no structures as massive as this lie along the lines of sight investigated here.

Upon excluding potential members outside of our redshift shell $\delta z$ and our maximum radius $\delta \theta$, we compute the velocity dispersion of the remaining group members. We calculate $\sigma_{\text {obs }}$ using the standard deviation, a clipped standard deviation, and the gapper algorithm (see Beers et al. [1990] for a comparison of these scale estimators):

$$
\sigma_{\text {obs,gapper }}=\frac{\sqrt{\pi}}{N(N-1)} \sum_{i}^{N-1} i(n-i)\left[c\left(z_{i+1}-z_{i-1}\right)\right] .
$$

Each of these methods resulted in the same group membership, and we report the final velocity dispersion obtained from the gapper algorithm due to the robustness of gap rank statistics for small samples (e.g., Beers et al. 1990) and to allow for direct comparison with the Wilman et al. (2005a) sample of groups. The updated value for $\sigma_{\text {obs }}$ obtained from the gapper estimate is then used to determine a new $\delta z$ and $\delta \theta$; this process is repeated until no more potential members are eliminated. For each potential group, stable membership was achieved in two iterations. Note that Wilman et al. (2005a) introduce a factor of 1.135 in their evaluation of $\sigma_{\text {obs, gapper }}$ to account for the $2 \sigma$ rejection in the membership-finding algorithm; because none of our candidate groups were affected by this criterion, we do not include the factor in our final estimate of $\sigma_{\text {obs }}$.

We retain any associations from our membership-finding algorithm with three or more members. This leaves us with four groups in the field of B1600 and four groups in the field of B2319. The details for these groups are listed in Table 3, and the velocity distribution of each group containing seven or more members is shown in Figure 3. Errors in the velocity dispersion were determined using a jackknife analysis; errors for groups with only three members are not meaningful. The group located at $z=0.5894$ in the field of B2319 contains the secondary lensing galaxy and could be two smaller groups interacting, as indicated by the bimodal velocity distribution, or it could be a small cluster. In this analysis we treat it as a single group.

\section{GROUP PROPERTIES}

As outlined in Table 3, we have detected three larger groups with seven or more members and five smaller groups. Whether many of these smaller groups are bound systems is questionable. For example, the two smallest groups associated with B1600 have significantly larger velocity dispersions than typical small groups (e.g., Merchán \& Zandivarez 2005). We report parameters for these small groups, but we limit our discussion to the three groups with at least seven members. The velocity dispersions of these larger groups are consistent with other moderate-redshift groups with comparable numbers of members (Wilman et al. 2005a; Momcheva et al. 2006; Mulchaey et al. 2006). In determining group parameters, we have assumed that the groups are relaxed, and we use the virial theorem to determine masses and radii.

We are hesitant to quote group properties due to the difficulty of obtaining full group memberships at these redshifts. Nevertheless, we provide estimates for the virial radius, mean pairwise separation, mass, and crossing time for each group; the values listed in Table 3 for these properties should be considered orderof-magnitude estimates. Following the definitions of Ramella et al. (1989), we define the group's virial radius

$$
R_{\mathrm{vir}}=\frac{\pi}{2} D_{\theta} N_{\text {pairs }}\left(\sum_{i} \sum_{j>i} \theta_{i j}^{-1}\right)^{-1}
$$
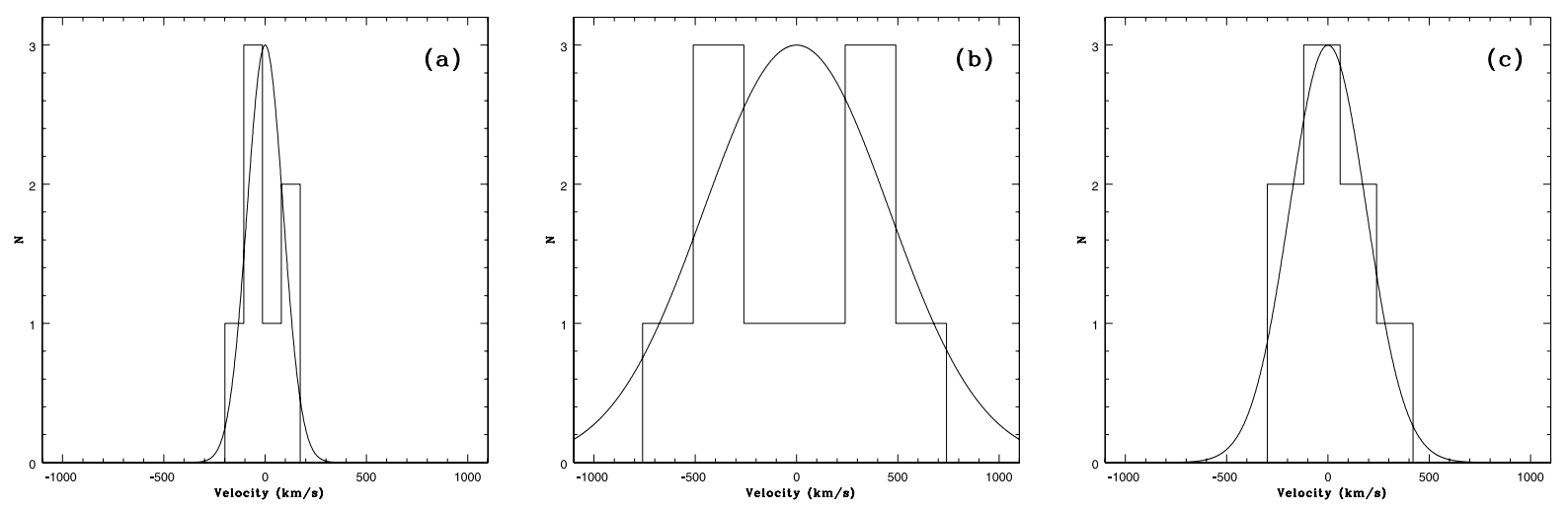

FIG. 3.- Velocity histograms for members of the groups associated with (a) B1600 and the (b) foreground and (c) background groups of B2319. 

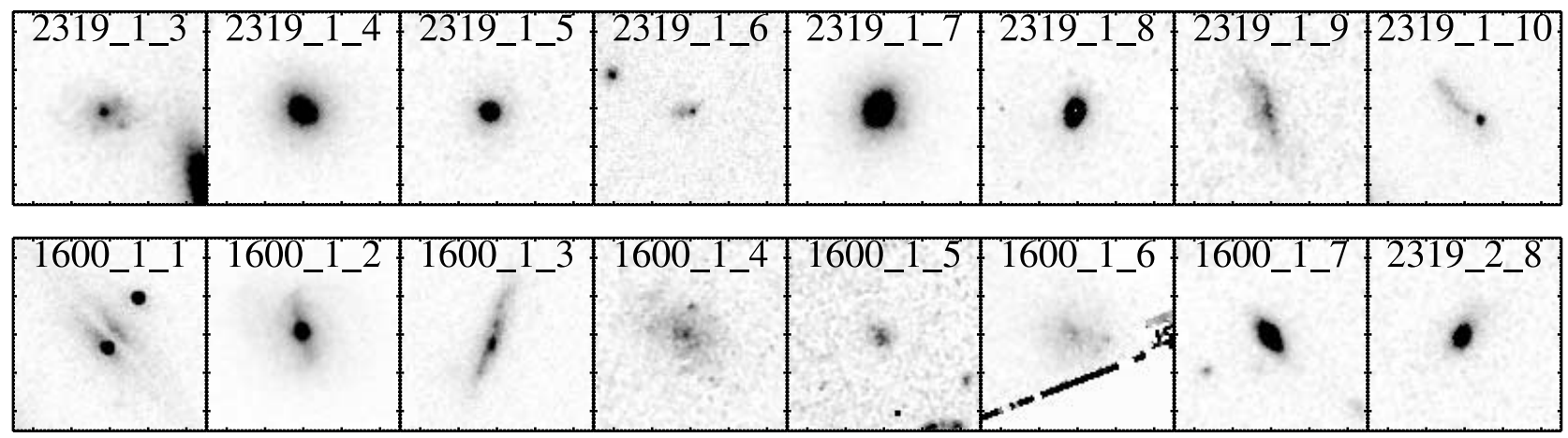

FIG. 4. - HST WFPC2 imaging of the B1600 and B2319 lens systems; each image is 5.05" on a side. The images for 1600_1_4 and 1600_1_5 are taken from the F555W filter, and the rest of the images are from the F814W filter. The lens in B1600 is 1600_1_1, and the secondary lensing galaxy for B2319 is 2319_1_10.

the mean (physical) separation between pairs

$$
R_{p}=\frac{4}{\pi} D_{\theta} N_{\text {pairs }}^{-1} \sum_{i} \sum_{j>i} \theta_{i j},
$$

the virial mass

$$
M_{\mathrm{vir}}=\frac{6 \sigma^{2} R_{\mathrm{vir}}}{G},
$$

and the crossing time

$$
t_{c}=\frac{3 R_{\mathrm{vir}} H_{0}}{5^{3 / 2} \sigma},
$$

where $D_{\theta}$ is the angular diameter distance to the group's redshift, $N_{\text {pairs }}=N_{\text {mem }}\left(N_{\text {mem }}-1\right) / 2$ is the number of unique group member pairs, $\theta_{i j}$ is the angular separation between group members, and $t_{c}$ is measured in Hubble times.

$H S T$ imaging is used to determine morphological classifications of group members that lie within the WFPC 2 field of view (Fig. 4), which includes most galaxies within $\sim 90^{\prime \prime}$ of the lensing galaxy. This includes all members of the B1600 group, eight members of the B2319 foreground group, and only one member of the B2319 background group. This final group lies at a greater distance from the lens system and is in the direction of the PC chip on WFPC2, causing a decreased field of view toward these galaxies. In our analysis we also indicate whether the group galaxies have strong emission lines present in their spectra. Considering the nature of the morphological and spectral features for each galaxy, we characterize the galaxies as either late- (spiral/ irregular morphologies or strong emission features and poor morphological information) or early-type galaxies (early-type or uncertain morphologies and no emission features).

We find that all of the galaxies that we associate with the B1600 group, including the lens galaxy, are late-type galaxies (see Table 4). With a velocity dispersion of $\approx 90 \mathrm{~km} \mathrm{~s}^{-1}$ and only seven members, this early-type fraction of zero is similar to some of the local universe groups studied by Zabludoff \& Mulchaey (1998), who found that groups with no early-type galaxies had lower velocity dispersions and fewer members than groups with nonzero earlytype fractions. Dai \& Kochanek (2005) do not find any X-ray emission associated with the B1600 group, which is also expected for groups with low velocity dispersions and low early-type fractions (e.g., Zabludoff \& Mulchaey 1998; Mulchaey et al. 2003). The upper limit on the group velocity dispersion from Dai \& Kochanek (2005) is $\sim 400 \mathrm{~km} \mathrm{~s}^{-1}$, and the velocity dispersion we have measured for the group is therefore consistent with the absence of observed X-ray emission.

The groups associated with B2319 have more varied populations than the B1600 group (see Tables 5 and 6). Using our characterization scheme, the early-type fractions for these groups are 0.5 and 0.63 for the foreground and background groups, respectively, although these results may be biased toward higher early-type fractions due to our selection criteria and our lack of high-resolution imaging for all group members. These earlytype fractions are typical of groups in the local universe that tend to also have X-ray emission associated with them (Zabludoff \& Mulchaey 1998; Mulchaey et al. 2003), although B2319 has not yet been observed at X-ray wavelengths. These early-type fractions lead us to believe that these two groups are likely to be bound and relaxed structures, and therefore the mass estimates

\begin{tabular}{|c|c|c|c|c|c|c|c|c|}
\hline Label & R.A. & Decl. & $z$ & $B^{\mathrm{a}}$ & $R^{\mathrm{a}}$ & $I^{\mathrm{a}}$ & Morphology ${ }^{\mathrm{b}}$ & Emission \\
\hline 1600_1_1_.................. & 160140.48 & 431648.0 & 0.4144 & 21.50 & 19.91 & 19.22 & $\mathrm{Sa}$ & Yes \\
\hline $1600-1-2 \ldots \ldots \ldots \ldots \ldots \ldots$ & 160140.84 & $43 \quad 1645.2$ & 0.4146 & 21.84 & 19.61 & 18.87 & $\mathrm{SBa}$ & Yes \\
\hline 1600_1_3 .................... & 160139.58 & 431648.3 & 0.4151 & 23.60 & 21.39 & 20.54 & $\mathrm{Sc}$ & Yes \\
\hline 1600_1_4.................... & 160142.83 & 431701.1 & 0.4140 & 23.60 & 21.96 & 21.53 & $\mathrm{Sd}$ & Yes \\
\hline 1600_1_5................... & 160141.83 & 431803.6 & 0.4149 & 25.36 & 24.04 & 23.76 & Irr & Yes \\
\hline 1600_1_6........................ & 160136.16 & $43 \quad 15 \quad 23.2$ & 0.4150 & 23.32 & 21.78 & 21.35 & Irr & Yes \\
\hline 1600_1_7 & 160137.70 & 431636.2 & 0.4144 & 24.03 & 21.87 & 21.13 & S0 & Yes \\
\hline
\end{tabular}

TABLE 4

Members of B1600 Group

Nоте.- Units of right ascension are hours, minutes, and seconds, and units of declination are degrees, arcminutes, and arcseconds.

${ }^{a}$ From Subaru Suprime-Cam imaging.

${ }^{\mathrm{b}}$ Determined from WFPC2 F555W and F814W imaging. 
TABLE 5

Members of B2319 Foreground Group

\begin{tabular}{|c|c|c|c|c|c|c|c|c|}
\hline Label & R.A. & Decl. & $z$ & $B^{\mathrm{a}}$ & $V^{\mathrm{a}}$ & $I^{\mathrm{a}}$ & Morphology ${ }^{b}$ & Emission \\
\hline 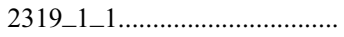 & 232137.25 & 052509.2 & 0.5867 & 24.28 & 23.61 & 21.85 & $\ldots$ & Yes \\
\hline 2319_1_2... & 232143.37 & 052543.9 & 0.5868 & 24.47 & 23.01 & 20.70 & $\ldots$ & No \\
\hline 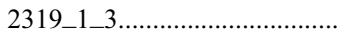 & 232137.54 & 052622.1 & 0.5893 & 23.27 & 22.39 & 20.39 & Irr & Yes \\
\hline 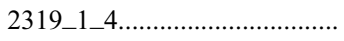 & 232138.24 & 052711.3 & 0.5861 & 23.96 & 22.09 & 19.59 & $\mathrm{E}$ & No \\
\hline 2319_1_5 ................................. & 232139.39 & 052719.2 & 0.5917 & 26.12 & 23.94 & 21.51 & $\mathrm{E}$ & Yes \\
\hline 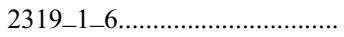 & 232140.73 & 052723.9 & 0.5922 & 24.24 & 23.94 & 22.75 & Irr & Yes \\
\hline 2319_1_7 & 232138.23 & 052738.3 & 0.5913 & 23.27 & 21.70 & 19.11 & $\mathrm{E}$ & No \\
\hline 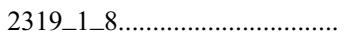 & 232138.41 & 052733.2 & 0.5876 & 24.97 & 23.12 & 20.45 & $\mathrm{E}$ & No \\
\hline 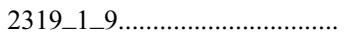 & 232138.82 & 052731.5 & 0.5910 & 25.73 & 24.47 & 22.31 & $\mathrm{Sa}$ & No \\
\hline 2319_1_10............................. & 232140.61 & 052738.2 & 0.5888 & 25.08 & 23.55 & 21.11 & Irr & Yes \\
\hline
\end{tabular}

NotE.-Units of right ascension are hours, minutes, and seconds, and units of declination are degrees, arcminutes, and arcseconds.

a From Keck LRIS imaging.

b Determined from WFPC2 F555W and F814W imaging when available.

derived from the measured velocity dispersions approximate the masses of these groups.

In Figure 5 we plot the locations of the early-type (circles) and late-type (squares) galaxies in each group with respect to the group center. Each field is $2 h^{-1} \mathrm{Mpc}$ on a side, and the central circle has a radius of $0.5 h^{-1} \mathrm{Mpc}$. We also show the location of the lensing galaxy, denoted by the dashed cross, with respect to the luminosity-weighted group centers. For the B2319 foreground group, we mark the galaxies with positive velocities relative to the mean group redshift (see Fig. $3 b$ ) with open symbols and galaxies with negative velocities with filled symbols.

The center of the B1600 group is coincident with the lensing galaxy, although the overall structure of the group is somewhat filamentary. There are several galaxies at a slightly higher redshift (including two galaxies at $z=0.419$; see Fig. 1), which might lend support to the idea that B1600 is embedded in a filament. The distribution of the inner five galaxies of the group is very compact, and the group meets all of the criteria of Hickson (1982) for compact groups except the requirement that the group be isolated from other galaxies (e.g., to distinguish compact groups from cluster - or in this case, group - cores).

We find that neither of the groups associated with B2319 is centered on the lensing galaxy. The background group has an elongated structure similar to the B1600 group. The B2319 groups do not show a preference for early-type galaxies lying in the center of the groups, contrary to what one might expect for relaxed groups. However, the foreground group appears to be quite compact, with seven members lying in a region with a radius of $\sim 100 h^{-1} \mathrm{kpc}$. In addition, there is not a clear separation between the positive velocity galaxies and the negative velocity galaxies (see Fig. $3 b$ ), indicating that the bimodality of the velocity distribution for the foreground group might be a result of incomplete sampling of faint group galaxies.

\section{LENS MODELS}

There are several ways to account for the group environments in the lensing models of B2319 and B1600. One method is to treat the group as a single dark matter halo with the galaxies in the group as massless test particles and to model the group as a smooth mass distribution. Alternatively, we could model each of the group members individually, ignoring any parent halo. This allows the possibility that our groups might be chance associations of galaxies instead of bound systems. Finally, we may model the group as lumpy masses embedded in a smooth distribution, a combination of the former two cases. This model of a halo with substructure is consistent with cluster observations and theoretical predictions (Natarajan \& Springel 2004; Gao et al. 2004; Zentner et al. 2005). However, due to incomplete information about our groups, in this analysis we only use the first two methods to give approximate indications for the contributions of the group environments to the lenses. We also create a sample of mock lenses in group environments using groups found in the SDSS database and evaluate the lensing contributions of the group halo and the individual galaxies using this mock catalog.

TABLE 6

Members of B2319 Background Group

\begin{tabular}{|c|c|c|c|c|c|c|c|c|}
\hline Label & R.A. & Decl. & $z$ & $B^{\mathrm{a}}$ & $V^{\mathrm{a}}$ & $I^{\mathrm{a}}$ & Morphology ${ }^{b}$ & Emission \\
\hline $2319 \_2 \_1 \ldots .$. & 232137.17 & 052557.2 & 0.6887 & 25.95 & 24.72 & 22.13 & $\ldots$ & No \\
\hline $2319 \_2 \_2 \ldots \ldots \ldots \ldots \ldots \ldots \ldots \ldots \ldots$ & 232139.98 & 052607.8 & 0.6891 & 23.97 & 23.69 & 22.65 & $\ldots$ & Yes \\
\hline 2319_2_3 .......................... & 232140.65 & 052627.7 & 0.6905 & 25.31 & 23.36 & 20.93 & $\cdots$ & No \\
\hline 2319_2_4 f............................. & 232141.99 & 052640.0 & 0.6881 & $23.79^{c}$ & $22.13^{\mathrm{c}}$ & $19.31^{\mathrm{c}}$ & $\ldots$ & No \\
\hline 2319_2_5 …..................... & 232141.99 & 052640.0 & 0.6884 & $\ldots{ }^{c}$ & $\ldots{ }^{\mathrm{c}}$ & $\ldots{ }^{c}$ & $\ldots$ & No \\
\hline 2319_2_6 ........................... & 232143.02 & 052648.7 & 0.6879 & 25.87 & 24.43 & 21.71 & $\ldots$ & No \\
\hline 2319_2_7 & 232141.97 & 052656.4 & 0.6903 & 25.05 & 24.45 & 22.82 & $\ldots$ & Yes \\
\hline 2319_2_8 ….................... & 232139.16 & 052829.3 & 0.6898 & 24.08 & 23.55 & 21.92 & S0 & Yes \\
\hline
\end{tabular}

Noте.-Units of right ascension are hours, minutes, and seconds, and units of declination are degrees, arcminutes, and arcseconds.

a From Keck LRIS imaging.

b Determined from WFPC2 F555W and F814W imaging when available.

${ }^{c}$ We are not able to extract individual magnitudes for these objects from our imaging data; however, the objects have distinct spectral traces, so we report them as individual galaxies. 

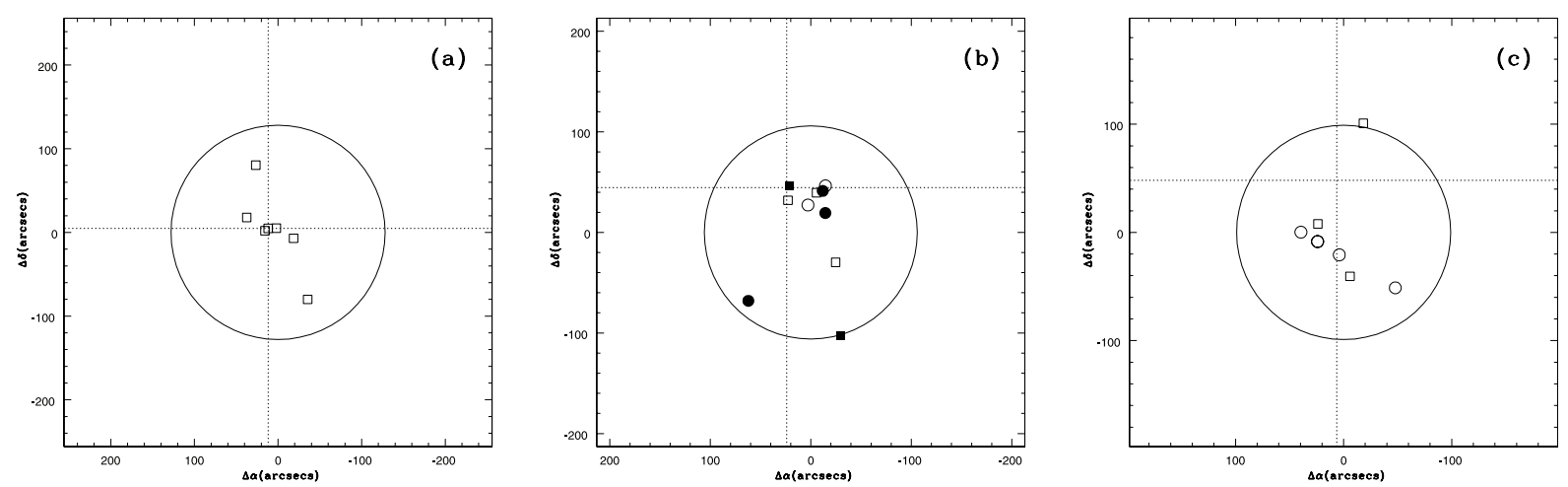

FIG. 5.- Spatial distribution of early-type (circles) and late-type (squares) galaxies for the (a) B1600 group and the (b) foreground and (c) background groups of B2319. The fields are centered on the group center and are $2 h^{-1} \mathrm{Mpc}$ on a side. The central circle has a radius of $0.5 h^{-1} \mathrm{Mpc}$, and the dashed cross marks the position of the lensing galaxy. The filled symbols in $(b)$ represent galaxies with negative velocities in the bimodal velocity distribution of Fig. $3 b$, and the open symbols represent galaxies with positive velocities.

For simplicity, we model all halos as SISs,

$$
\kappa_{\mathrm{SIS}}(\theta)=\frac{0.9 D_{l s} \sigma_{250}^{2}}{D_{s} \theta}
$$

where $\kappa_{\text {SIS }}$ is the convergence, $D_{l s}$ is the angular diameter distance between the lens and source, $D_{s}$ is the angular diameter distance to the source plane, $\theta$ is the distance to the centroid of the SIS measured in arcseconds, and $\sigma_{250}$ is the velocity dispersion measured in units of $250 \mathrm{~km} \mathrm{~s}^{-1}$. The magnitude of the shear for an SIS model is the same as the convergence, $\gamma_{\text {SIS }}=\kappa_{\text {SIS }}$, and the orientation of the shear is along the axis joining the center of the SIS and the point at which the shear is being evaluated. For our individual-galaxies model, we assign SIS velocity dispersions to each galaxy by assuming that an $L_{*}$ galaxy has a fiducial velocity dispersion of $\sigma_{*}=(185,205,225) \mathrm{km} \mathrm{s}^{-1}$ (for spiral, lenticular, and elliptical galaxies, respectively, motivated by Fukugita $\&$ Turner 1991) and the velocity dispersions of the group galaxies are scaled as

$$
\frac{L}{L_{*}}=\left(\frac{\sigma}{\sigma_{*}}\right)^{\gamma}
$$

where $\gamma=3.1$ for spiral galaxies and $\gamma=4$ for E and S0 galaxies. The total shear is determined by forming $\gamma_{1}$ and $\gamma_{2}$ (e.g., Narayan \& Bartelmann 1999) for each galaxy, summing these terms from all group members, and then determining the magnitude and orientation of the total shear from the $\gamma_{1, \text { total }}$ and $\gamma_{2, \text { total }}$ components. For the group model, we use the luminosityweighted average position of the group galaxies to determine the group centroid.

\subsection{B2319 Lens Models}

Previous lens models for B2319 were found to inadequately reproduce the image configuration without applying a large shear term $\left(\gamma=0.14\right.$, P.A. $\gamma=-22^{\circ}$; Rusin et al. 2001). The necessary shear is essentially perpendicular to the expected orientation of the shear caused by the lensing galaxy, and this excess shear is therefore assumed to be caused by another object. We investigate whether the foreground and background groups we have detected can adequately account for this discrepancy. Although the groups associated with B2319 are not at the same redshift as the primary lensing galaxy, we calculate the shear at the lensing plane of the groups and identify this as the same shear required by Rusin et al. (2001; see also Fassnacht et al. 2006 and Momcheva et al. 2006).

We create two models for the system by accounting for the foreground and background groups and for the individual galaxies of both groups. The results for these models are collected in Table 7. We find a total additional shear of $\gamma=0.04$ at a position angle (P.A.) of $46^{\circ}$ for the group halo model and a shear of $\gamma=0.04$ at a P.A. of $-57^{\circ}$ for the individual-galaxies model. Both models differ substantially in both magnitude and orientation from the shear required by Rusin et al. (2001). In addition to the secondary lensing galaxy (2319_1_10), there is a faint galaxy $\sim 2$ " southeast of the lensing galaxy that is visible in our HST imaging (see Fig. 6). This galaxy lies approximately along the shear axis required by Rusin et al. (2001) (the P.A. is approximately $-40^{\circ}$ ), and although it is probably not very massive, this third galaxy might provide an important contribution to the lensing due to its proximity to the lens.

\subsection{B1600 Lens Models}

The model for B1600 adequately reproduces the observed image distribution with physically reasonable parameters (Koopmans et al. 1998). However, the presence of the group that includes the lensing galaxy adds additional mass to the lens plane. If the scale of the mass distribution is much larger than the image separation and the group centroid is far enough from the lens system, then the group can be approximated as an infinite mass sheet. This mass sheet increases the convergence in the lens model, which in turn affects the value of $H_{0}$ determined for this lens system (see $\S 6.3$ ). Note that the group is not a true mass sheet and also adds a small shear to the model. However, lens models tend to accurately account for all shear present, and in this sense the shear from the

TABLE 7

B2319 Lens Environment Modeling

\begin{tabular}{rcccccccrr}
\hline \hline Model & $\kappa_{\text {frg }}$ & $\gamma_{\text {frg }}$ & P.A.frg & $\kappa_{\text {bkg }}$ & $\gamma_{\text {bkg }}$ & P.A.bkg & $\kappa_{\text {total }}$ & $\gamma_{\text {total }}$ & P.A.total \\
\hline Groups ............................... & 0.038 & 0.038 & -132 & 0.004 & 0.004 & 170 & 0.042 & 0.036 \\
Individual galaxies ................ & 0.102 & 0.042 & -73 & 0.025 & 0.022 & -15 & 0.127 & 0.038 & -57 \\
\hline
\end{tabular}

NoтES._Values marked "frg" are for the foreground group, those marked "bkg" are for the background group, and those marked "total " are for the sum of the contributions of the foreground and background groups. 


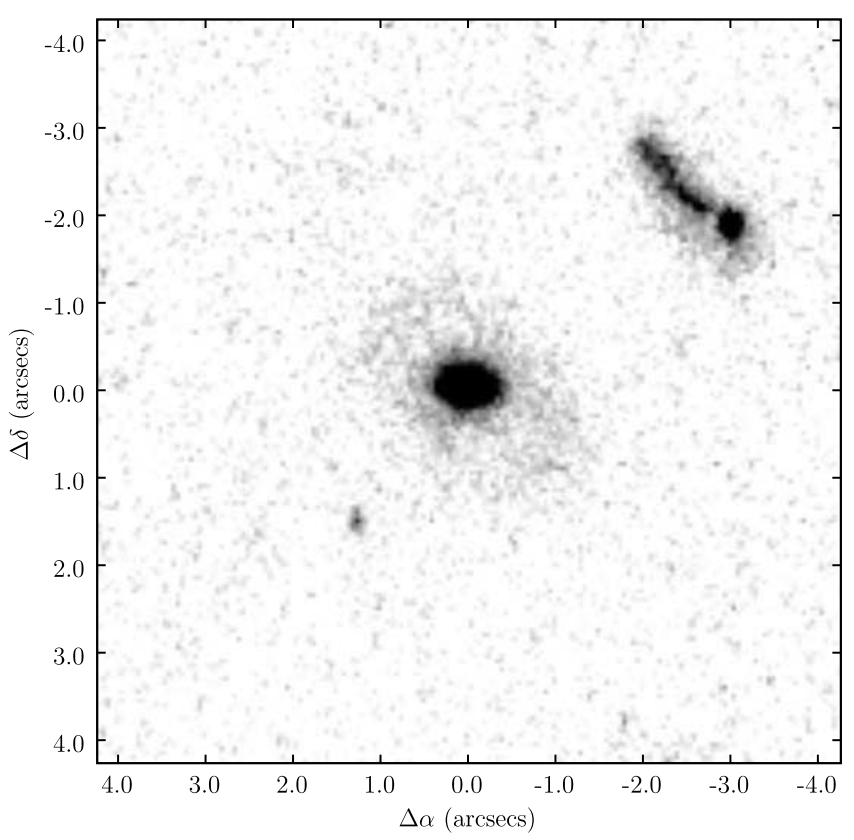

FIG. 6.- $-H S T$ WFPC2 F814W imaging of B2319. The secondary perturber is $\sim 3.4^{\prime \prime}$ northwest of the lens, and the third perturber is $\sim 2^{\prime \prime}$ southeast of the lensing galaxy.

B1600 group is not additional shear but rather a reaccounting of the shear (by removing part of the shear contribution from the lens galaxy, for example). In this analysis we calculate the expected added convergence due to the group, the individual galaxies excluding the lensing galaxy, and the individual galaxies excluding the lens and its neighbor galaxy, 1600_1_2. The neighbor galaxy has already been included in some lens models (see, e.g., Maller et al. 2000), and our observations now confirm that this galaxy is at the same redshift as the lens. Results for the group lens models are collected in Table 8. Previous analyses of B1600 have derived values of $H_{0}$ between 52 and $60 \mathrm{~km} \mathrm{~s}^{-1}$ $\mathrm{Mpc}^{-1}$ (Burud et al. 2000; Koopmans et al. 2000), already lower than the values determined by other methods (e.g., Sánchez et al. 2006; Tegmark et al. 2004; Freedman et al. 2001). The additional convergence due to the environment of B1600 lowers these values by a further $\sim 10 \%$ if the galaxies are considered individually, although most of this contribution is from the closest neighbor to the lens (see Table 8). This effect is already seen in the different values for $H_{0}$ determined by Burud et al. (2000) compared to Koopmans et al. (2000); Burud et al. (2000) include the neighbor and derive a lower value for $H_{0}$ than Koopmans et al. (2000), who do not include the neighboring galaxy.

\subsection{SDSS Group Models}

We have used the SDSS DR5 (Adelman-McCarthy et al. 2007) spectroscopic catalog to expand the group membership of a

TABLE 8

B1600 Lens Environment Modeling

\begin{tabular}{|c|c|c|c|}
\hline Model & $\kappa_{\text {env }}$ & $\gamma_{\mathrm{env}}$ & P.A.env \\
\hline Group ........ & 0.015 & 0.015 & -158 \\
\hline Individual galaxies ${ }^{\mathrm{a}} .$. & 0.113 & 0.092 & -61 \\
\hline Individual galaxies ${ }^{\mathrm{b}}$ & 0.025 & 0.021 & 82 \\
\hline
\end{tabular}

\footnotetext{
${ }^{\text {a }}$ Excluding the lensing galaxy.

$\mathrm{b}$ Excluding the lensing galaxy and its neighbor, 1600_1_2. Note that most of the contribution in the individual-galaxies model comes from the galaxy closest to the lens.
}

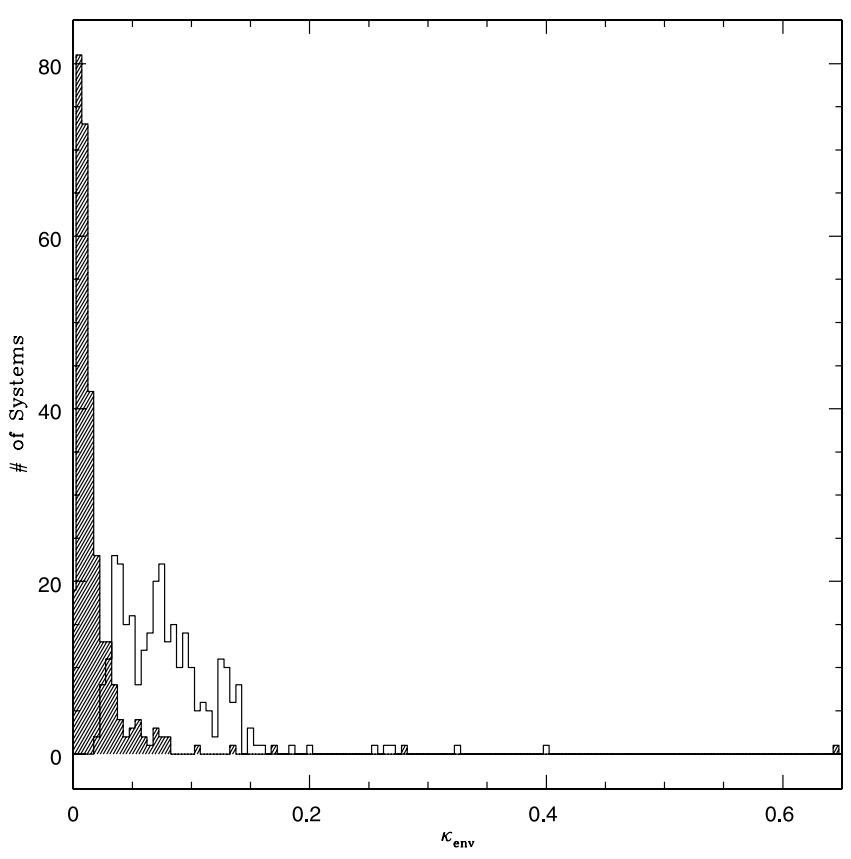

Fig. 7.-Environmental convergence provided by group halos (shaded areas) and neighboring galaxies (unshaded areas) from our SDSS group models.

sample of 56 isolated groups at low redshifts $(0.007<z<0.04)$ originally identified in the catalog of Merchán \& Zandivarez (2005). We select groups with velocity dispersions of 150 $450 \mathrm{~km} \mathrm{~s}^{-1}$, greater than 30 members (to accurately determine the velocity dispersion and group centroid), and 8 or fewer $r<m^{*}$ members to match the properties of typical lens groups. Velocity dispersions are calculated using the biweight estimate of scale (due to the large numbers of members, e.g., Beers et al. 1990). We project these groups to a fiducial lens redshift of $z_{\text {lens }}=0.35$ and create a total of 300 mock lens systems by considering each bright $\left(r<m^{*}+0.5\right)$ group member to be a potential lens. All of our models assume a source redshift of $z_{\text {source }}=1.5$, and we calculate

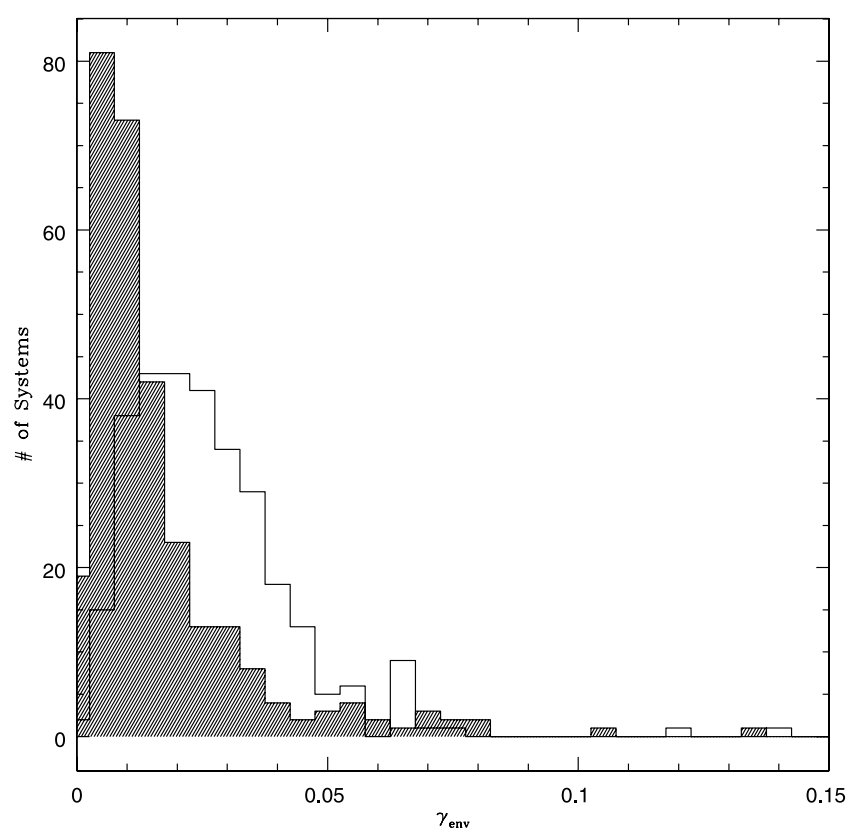

FIG. 8.- Magnitude of the shear contributed by common group halos (shaded areas) and neighboring galaxies (unshaded areas) from our SDSS group models. The extreme values for the group shear seen in Fig. 7 have been trimmed to better show the separation of the two distributions. 


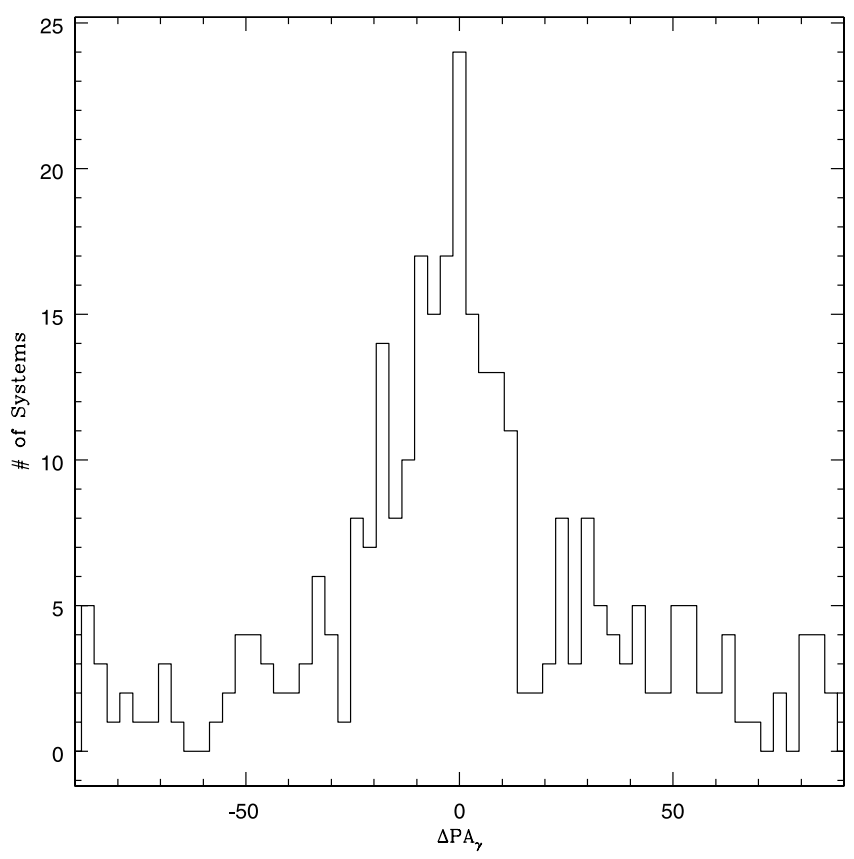

Fig. 9.-Difference in P.A. of the shears for the common group halo model and the individual-galaxies model.

the effects of the environments due to the group halos and the individual members. Figures 7 and 8 show the distributions of $\kappa_{\text {env }}$ and $\gamma_{\mathrm{env}}$ for the group (shaded areas) and individual-galaxy models. Figure 9 shows the difference in P.A. of the environmental shear between the group model and the individual-galaxy model for each system.

The median convergence for the group halo model is $\kappa_{\mathrm{env}}=$ 0.010 , and the median convergence from the individual-galaxies model is $\kappa_{\text {env }}=0.072$. The median magnitude of the shear contributed by the individual galaxies is $\gamma_{\text {env }}=0.023$, only about 2 times higher than $\gamma_{\mathrm{env}}=0.010$ for the group halo model. The individual-galaxy model shear P.A.s tend to follow the group halo model P.A.s, although with considerable spread. The group halo model shows a tail of high convergence values; these systems represent models for the brightest group galaxies, which tend to be located near the center of the group halo (although not every brightest group galaxy lens model has a large contribution from the group halo). It is also worth noting that the individualgalaxy model has a minimum convergence of $\kappa=0.019$, nearly twice the value of the median group halo contribution.

\section{DISCUSSION}

\subsection{Are These Groups Real?}

The absence of early-type galaxies and X-ray emission indicates that the B1600 group may not be a relaxed or bound system. Furthermore, the elongated structure of the group may favor this unrelaxed interpretation, although groups have been shown to have a wide range of morphologies (Zabludoff \& Mulchaey 1998; Wilman et al. 2005a). Although our sampling is not deep enough to make strong statements regarding the characteristic length and mass scales for this group, we find the mass to be consistent with other groups with similar membership (e.g., Zabludoff \& Mulchaey 1998; Ramella et al. 1989). Williams et al. (2006) find evidence for a red sequence of galaxies centered approximately $1^{\prime}$ east of the B1600 group with a suggested red$\operatorname{shift} z \sim 0.5$. This structure is not apparent in our spectroscopic data (see Table 3), although our selection criteria might have biased us away from selecting early-type galaxies lying on a red sequence.

The arguments for and against the bound interpretation of the B1600 group all rely on comparisons with local groups. It is perhaps reasonable to expect that groups at higher redshifts are dynamically younger and therefore their constituent galaxies have not had time to undergo processing, although the dynamical times listed in Table 3 are short. This would account for the absence of early-type galaxies and hot intragroup gas. The compactness of the distribution of the central members of the group and the presence of a strong gravitational lens are indicators that, at a minimum, the central structure of the group is bound in a common halo, and we therefore conclude that B1600 lies in a group environment.

The groups associated with B2319 are similar to groups found in the local universe; the numbers of members, early-type fractions, and velocity dispersions of these two groups are typical of local groups. The foreground group is potentially a poor cluster and is a good candidate for X-ray follow-up (e.g., Jeltema et al. 2006). The nature of the background group is more difficult to discern; the group has an irregular morphology with no proper core but a large early-type fraction. The absence of a core might indicate that the group is forming, while the large early-type fraction suggests the constituent galaxies have had several significant interactions. It is worth noting that we did not target galaxies at a redshift of $z \sim 0.7$, and deeper spectroscopy of the field might make a core structure more apparent for the group. In any case, the number of members (eight) and the approximately Gaussian nature of the velocity histogram for this group (Fig. 3c) indicate that this is a group associated with a common halo.

\subsection{Group Lens Models}

It is very difficult to create accurate lensing models of moderateredshift groups. The lens corrections based on individual galaxies rely on incomplete membership information and incorrect modeling of velocity dispersions for individual group members. These problems are probably less severe than those present for the group halo model. Velocity dispersion estimates for group halos can be off by significant amounts because of incomplete group membership sampling (perhaps by as much as a factor of 1.5-3; e.g., Zabludoff \& Mulchaey 1998), and tens of redshifts are necessary to hope to get an accurate estimate. Due to the quadratic dependence of SIS models on velocity dispersions, modeling errors for SIS models can be substantial for poorly sampled groups. Unfortunately, it is very difficult to obtain redshifts for $m>m^{*}+2$ galaxies at moderate redshifts, and highly accurate group velocity dispersions cannot be obtained for most moderateredshift lens systems residing in poor groups. In addition, different centroiding methods can yield very different angular offsets of the group from the lens due to sampling small numbers of the group members (e.g., Fassnacht \& Lubin 2002; Fassnacht et al. 2006). Finally, the SIS model assumes a relaxed group halo, although this might not be the case for many moderate-redshift groups (see the discussion of the B1600 group above).

Deep X-ray observations of lens systems would potentially correct for all of the problems associated with modeling group halos; the presence of diffuse X-ray emission would indicate the presence of a group halo, the X-ray temperature could be used as a mass proxy, and the diffuse X-ray centroid would be a good estimate of the mass centroid (Mulchaey et al. 2003; Mulchaey \& Zabludoff 1998). However, for all but the most massive groups, it is very difficult to detect intragroup X-ray emission due to the low luminosities and cosmological dimming of these moderateredshift sources (Dai \& Kochanek 2005; C. D. Fassnacht et al. 
2007, in preparation), although some detections have been made (Jeltema et al. 2006; Mulchaey et al. 2006; Grant et al. 2004).

Fortunately, our modeling of the SDSS groups indicates that the group halo generally has only a very small effect on the lensing. With the exception of lensing by brightest group galaxies that might be lying at the center of the group potential, nearly $90 \%$ of the environmental contribution to the convergence is due to neighboring galaxies. This is consistent with our observational findings for B1600 and B2319, as shown in Tables 7 and 8 . The greater contribution of the individual galaxies compared to the group halo is also consistent with the effect found by Momcheva et al. (2006), although those authors found the magnitudes of the group halo and individual galaxy contributions to be more comparable.

The total additional convergence from the environment is dominated by individual galaxy halos, and it is important that these be modeled accurately. Here we have assumed isothermal profiles for all galaxies, ignoring truncation. However, the truncation cutoff radius for galaxies in cluster environments is typically between 20 and $50 \mathrm{kpc}$ (Limousin et al. 2007), while truncation in field galaxies is much larger. Group galaxies probably lie somewhere between these extremes, so truncation may not be a significant problem. Furthermore, any galaxies that strongly contribute to the lensing are within $\sim 15^{\prime \prime}$ of the lens, which is within the cutoff radius for cluster galaxies. The convergence due to a $\sigma=160 \mathrm{~km} \mathrm{~s}^{-1}$ galaxy $10^{\prime \prime}$ from a lens at redshift $z=0.35$ and a source at $z=1.5$ is $\kappa=0.025$, while the convergence of a similar galaxy $30^{\prime \prime}$ away drops to less than 0.01 . Thus, any galaxies with a nonnegligible environmental contribution do not need to be modeled as truncated halos. However, our model still has an uncertainty associated with assigning velocity dispersions to galaxies. Nevertheless, the method we have applied here is probably the appropriate approach to constraining $\kappa_{\text {env }}$, as this method also can account for significant secondary lenses that are not at the primary lens redshift (e.g., see the "suspension bridge" diagrams of Momcheva et al. 2006).

\section{3. $H_{0}$ Implications}

The additional convergence due to a uniform mass sheet will systematically decrease the value of $H_{0}$ compared to models that do not account for the lens environment,

$$
H_{0, \text { true }}=H_{0, \text { meas }}\left(1-\kappa_{\text {env }}\right),
$$

where $\kappa_{\text {env }}$ represents the uniform mass sheet (see $\S 13.1 .1$ of Schneider et al. 1992). The value of $\kappa_{\text {env }}$ is always greater than zero, and the true value of $H_{0}$ will be necessarily (although perhaps negligibly) lower than the value estimated from lensing. This is one consequence of the well-known lensing "mass sheet degeneracy" (e.g., Falco et al. 1985). This degeneracy can be broken if multiple sources at different redshifts are lensed or if a stellar velocity dispersion is determined for the lens system, allowing the mass slope to be directly measured. In the absence of this information, an estimate of $\kappa_{\text {env }}$ must be obtained separately by investigating the environment of the lens system. For B1600, the contribution to $\kappa_{\text {env }}$ from neighboring galaxies suggests a $10 \%$ decrease in the determined value of $H_{0}$ for models that did not include any information about the environment (Koopmans et al. 2000 ), or a $3 \%$ decrease for models that included the nearest galaxy (Burud et al. 2000). Because the group halo contribution to $\kappa_{\text {env }}$ is very small, our uncertainty of whether B1600 lies in a bound, relaxed group (i.e., with a common group halo) is relatively unimportant in determining the perturbations to $H_{0}$; for $\mathrm{B} 1600$, the assumed group halo only changes $H_{0}$ by $\approx 1 \%$. Note that ignoring lens environments causes a systematically inflated value of $H_{0}$, and our analysis simply removes (some of) this bias; this should be considered a reinterpretation of the previously published values of $H_{0}$ for this lens system and not a new calculation for the Hubble constant.

\subsection{Environment and Shear}

We find that the environment adds a small contribution to the shear, although we emphasize that this is very dependent on the membership determined for the groups and that most of the shear contribution is already accounted for in two-image lens models. In this sense, an investigation of the environment provides information that describes the cause of the shear, breaking the degeneracy between mass ellipticity and environment. Our models for the B2319 group are unable to reproduce the shear expected by the original lens models (Rusin et al. 2001). This may indicate that the lens is responsible for part of the shear but its parent halo is not aligned with the luminous content of the galaxy. We note that the orientation of the lens galaxy central bulge is offset from the disk orientation (see Fig. 6). Furthermore, a detailed model of both the faint and bright lens neighbors might account for most of the external shear, with the foreground and background groups having negligible effects.

Our modeling of the SDSS groups indicates that most lens systems that require large external shears will either have a very close companion galaxy or will be brightest group galaxies located very near the group center (this is the case for all systems in the tails of the distributions of Fig. 8). The B2319 system and other recent studies (e.g., McKean et al. 2007) indicate that this shear may be caused by faint companion galaxies very close to the lens, underscoring the importance of fully investigating the local environments of lens systems.

\section{CONCLUSIONS}

The strong lens systems B1600+434 and B2319+051 are both associated with group structures, giving further evidence that strong lenses may provide an efficient means of identifying groups at moderate redshifts. The group properties are consistent with groups in the local universe, although we will make a more detailed comparison between local and moderate-redshift groups on completing our survey of lens environments. The two systems presented here increase the number of spectroscopically confirmed groups associated with lenses by $\approx 20 \%$, although it is still unclear how many lens systems in general are associated with groups. The significant contributions of lens environments to lens models found here and by Momcheva et al. (2006) indicate that accurate environment models are necessary for obtaining precision measurements with lensing. For example, the total contribution of lens environments to the lensing convergence is large enough to cause systematic biases of $\approx 10 \%$ to derivations of $H_{0}$ from lensing.

The environmental contributions to the lens models of B2319 and B1600 are dominated by the galaxies with small separations from the lensing galaxy, and this indicates that the environments of most lenses can be quantified by investigating the individual galaxies within $\sim 15^{\prime \prime}$ of the lenses. This will minimize the amount of telescope time needed to quantify $\kappa_{\text {env }}$ and will also account for galaxies not at the same redshift as the lens galaxy. The exception to this is when the lensing galaxy is the brightest group galaxy and therefore is expected to be close to the center of the group halo. The group halo may provide a significant additional convergence for these lenses, although it is difficult to accurately describe the lensing effects of the group halo. In general, ignoring the group halo results in a systematic error in 
determining $\kappa_{\text {env }}$, but this error is small compared to the uncertainties in determining the properties of the group halo.

Based in part on observations made with the NASA/ESA Hubble Space Telescope, obtained from the Data Archive at the Space Telescope Science Institute (STScI). STScI is operated by the Association of Universities for Research in Astronomy, Inc., under NASA contract NAS5-26555. These observations are associated with program AR-10300, supported by NASA through a grant from STScI. In addition, some of the data presented herein were obtained at the W. M. Keck Observatory, which is operated as a scientific partnership among the California Institute of Technology, the University of California, and the Na- tional Aeronautics and Space Administration. The Observatory was made possible by the generous financial support of the W. M. Keck Foundation. The authors wish to recognize and acknowledge the very significant cultural role and reverence that the summit of Mauna Kea has always had within the indigenous Hawaiian community. We are most fortunate to have the opportunity to conduct observations from this mountain. This work is also based in part on data collected at the Subaru Telescope and obtained from the SMOKA science archive at the Astronomical Data Analysis Center, which are operated by the National Astronomical Observatory of Japan. Part of this work was supported by the European Community's Sixth Framework Marie Curie Research Training Network Programme, Contract No. MRTN-CT-2004-505183 “ANGLES.”
Aarseth, S. J., \& Fall, S. M. 1980, ApJ, 236, 43

Abazajian, K., et al. 2005, ApJ, 625, 613

Aceves, H., \& Velázquez, H. 2002, Rev. Mex. AA, 38, 199

Adelman-McCarthy, J., et al. 2007, ApJS, submitted

Baba, H., et al. 2002, in ASP Conf. Ser. 281, Astronomical Data Analysis Software and Systems XI, ed. D. A. Bohlender, D. Durand, \& T. H. Handley (San Francisco: ASP), 298

Bahcall, J. N., Kirhakos, S., Saxe, D. H., \& Schneider, D. P. 1997, ApJ, 479, 642

Balogh, M., et al. 2004, MNRAS, 348, 1355

Beers, T. C., Flynn, K., \& Gebhardt, K. 1990, AJ, 100, 32

Best, P. N. 2004, MNRAS, 351, 70

Best, P. N., Kauffmann, G., Heckman, T. M., Brinchmann, J., Charlot, S., Ivezić, Ž., \& White, S. D. M. 2005, MNRAS, 362, 25

Blandford, R., Surpi, G., \& Kundić, T. 2001, in ASP Conf. Ser. 237, Gravitational Lensing: Recent Progress and Future Goals, ed. T. G. Brainerd \& C. S. Kochanek (San Francisco: ASP), 65

Bower, R. G., \& Balogh, M. L. 2004, in Clusters of Galaxies: Probes of Cosmological Structure and Galaxy Evolution, ed. J. S. Mulchaey, A. Dressler, \& A. Oemler (Cambridge: Cambridge Univ. Press), 325

Browne, I. W. A., et al. 2003, MNRAS, 341, 13

Burud, I., et al. 2000, ApJ, 544, 117

Carlberg, R. G., Yee, H. K. C., Morris, S. L., Lin, H., Hall, P. B., Patton, D. R., Sawicki, M., \& Shepherd, C. W. 2001a, ApJ, 552, 427

. 2001b, ApJ, 563, 736

Coil, A. L., et al. 2004, ApJ, 609, 525 2006, ApJ, 638, 668

Colless, M., et al. 2001, MNRAS, 328, 1039

Collister, A. A., \& Lahav, O. 2005, MNRAS, 361, 415

Conselice, C. J., Bershady, M. A., Dickinson, M., \& Papovich, C. 2003, AJ, 126,1183

Dai, X., \& Kochanek, C. S. 2005, ApJ, 625, 633

Dalal, N., \& Watson, C. R. 2004, preprint (astro-ph/040948)

D’Onghia, E., \& Lake, G. 2004, ApJ, 612, 628

Evrard, A. E., et al. 2002, ApJ, 573, 7

Falco, E. E., Gorenstein, M. V., \& Shapiro, I. I. 1985, ApJ, 289, L1

Fassnacht, C. D., Gal, R. R., Lubin, L. M., McKean, J. P., Squires, G. K., \& Readhead, A. C. S. 2006, ApJ, 642, 30

Fassnacht, C. D., \& Lubin, L. M. 2002, AJ, 123, 627

Faure, C., Alloin, D., Kneib, J. P., \& Courbin, F. 2004, A\&A, 428, 741

Freedman, W. L., et al. 2001, ApJ, 553, 47

Fukugita, M., \& Turner, E. L. 1991, MNRAS, 253, 99

Gao, L., White, S. D. M., Jenkins, A., Stoehr, F., \& Springel, V. 2004, MNRAS, 355, 819

Gerke, B. F., et al. 2005, ApJ, 625, 6

Grant, C. E., Bautz, M. W., Chartas, G., \& Garmire, G. P. 2004, ApJ, 610, 686

Hickson, P. 1982, ApJ, 255, 382

Hoekstra, H., et al. 2001, ApJ, 548, L5

Jackson, N., et al. 1995, MNRAS, 274, L25

Jeltema, T. E., Mulchaey, J. S., Lubin, L. M., Rosati, P., \& Böhringer, H. 2006, ApJ, 649, 649

Kauffmann, G., White, S. D. M., Heckman, T. M., Ménard, B., Brinchmann, J., Charlot, S., Tremonti, C., \& Brinkmann, J. 2004, MNRAS, 353, 713

Keeton, C. R., Christlein, D., \& Zabludoff, A. I. 2000, ApJ, 545, 129

Keeton, C. R., \& Zabludoff, A. I. 2004, ApJ, 612, 660

Kochanek, C. S. 2002, ApJ, 578, 25

2003, ApJ, 583, 49

\section{REFERENCES}

Kochanek, C. S., \& Schechter, P. L. 2004, in Measuring and Modeling the Universe, ed. W. L. Freedman (Cambridge: Cambridge Univ. Press), 117

Koopmans, L. V. E., de Bruyn, A. G., \& Jackson, N. 1998, MNRAS, 295, 534

Koopmans, L. V. E., de Bruyn, A. G., Xanthopoulos, E., \& Fassnacht, C. D. 2000, A\&A, 356, 391

Koopmans, L. V. E., Treu, T., Fassnacht, C. D., Blandford, R. D., \& Surpi, G. 2003, ApJ, 599, 70

Kundic, T., Cohen, J. G., Blandford, R. D., \& Lubin, L. M. 1997a, AJ, 114, 507

Kundic, T., Hogg, D. W., Blandford, R. D., Cohen, J. G., Lubin, L. M., \& Larkin, J. E. 1997b, AJ, 114, 2276

Lehár, J., et al. 2000, ApJ, 536, 584

Limousin, M., Kneib, J. P., Bardeau, S., Natarajan, P., Czoske, O., Smail, I., Ebeling, H., \& Smith, G. P. 2007, A\&A, 461, 881

Lin, L., et al. 2004, ApJ, 617, L9

Lubin, L. M., Fassnacht, C. D., Readhead, A. C. S., Blandford, R. D., \& Kundić, T. 2000, AJ, 119, 451

Maller, A. H., Simard, L., Guhathakurta, P., Hjorth, J., Jaunsen, A. O., Flores, R. A., \& Primack, J. R. 2000, ApJ, 533, 194

McKean, J. P., et al. 2007, MNRAS, 378, 109

McLure, R. J., \& Dunlop, J. S. 2001, MNRAS, 321, 515

Merchán, M. E., \& Zandivarez, A. 2005, ApJ, 630, 759

Miller, C. J., Nichol, R. C., Gómez, P. L., Hopkins, A. M., \& Bernardi, M. 2003, ApJ, 597, 142

Miyazaki, S., et al. 2002, PASJ, 54, 833

Möller, O., Natarajan, P., Kneib, J.-P., \& Blain, A. W. 2002, ApJ, 573, 562

Momcheva, I., Williams, K., Keeton, C., \& Zabludoff, A. 2006, ApJ, 641, 169

Morgan, N. D., Kochanek, C. S., Pevunova, O., \& Schechter, P. L. 2005, AJ, 129,2531

Mulchaey, J. S., Davis, D. S., Mushotzky, R. F., \& Burstein, D. 2003, ApJS, 145,39

Mulchaey, J. S., Lubin, L. M., Fassnacht, C. D., Rosati, P., \& Jeltema, T. E. 2006, ApJ, 646, 133

Mulchaey, J. S., \& Zabludoff, A. I. 1998, ApJ, 496, 73

Myers, S. T., et al. 2003, MNRAS, 341, 1

Narayan, R., \& Bartelmann, M. 1999, in Formation of Structure in the Universe, ed. A. Dekel \& J. P. Ostriker (Cambridge: Cambridge Univ. Press), 360

Natarajan, P., \& Springel, V. 2004, ApJ, 617, L13

Oguri, M., Keeton, C. R., \& Dalal, N. 2005, MNRAS, 364, 1451

Oke, J. B., et al. 1995, PASP, 107, 375

Ouchi, M., et al. 2004, ApJ, 611, 660

Padilla, N. D., et al. 2004, MNRAS, 352, 211

Patton, D. R., et al. 2002, ApJ, 565, 208

Ramella, M., Geller, M. J., \& Huchra, J. P. 1989, ApJ, 344, 57

Reed, D., Governato, F., Quinn, T., Gardner, J., Stadel, J., \& Lake, G. 2005 , MNRAS, 359, 1537

Rusin, D., et al. 2001, AJ, 122, 591

Sánchez, A. G., Baugh, C. M., Percival, W. J., Peacock, J. A., Padilla, N. D., Cole, S., Frenk, C. S., \& Norberg, P. 2006, MNRAS, 366, 189

Schneider, P., Ehlers, J., \& Falco, E. 1992, Gravitational Lenses (Berlin: Springer)

Sheinis, A. I., Bolte, M., Epps, H. W., Kibrick, R. I., Miller, J. S., Radovan, M. V., Bigelow, B. C., \& Sutin, B. M. 2002, PASP, 114, 851

Tegmark, M., et al. 2004, Phys. Rev. D, 69, 103501

Tonry, J. L. 1998, AJ, 115, 1

Tonry, J. L., \& Kochanek, C. S. 1999, AJ, 117, 2034 - 2000, AJ, 119, 1078 
Tully, R. B. 1987, ApJ, 321, 280

Weinmann, S. M., van den Bosch, F. C., Yang, X., \& Mo, H. J. 2006, MNRAS, 366,2

Williams, K. A., Momcheva, I., Keeton, C. R., Zabludoff, A. I., \& Lehár, J. 2006, ApJ, 646, 85

Wilman, D. J., Balogh, M. L., Bower, R. G., Mulchaey, J. S., Oemler, A., Carlberg, R. G., Morris, S. L., \& Whitaker, R. J. 2005a, MNRAS, 358, 71
Wilman, D. J., et al. 2005b, MNRAS, 358, 88

Yang, X., Mo, H. J., Jing, Y. P., van den Bosch, F. C., \& Chu, Y. 2004, MNRAS, 350, 1153

York, D. G., et al. 2000, AJ, 120, 1579

Zabludoff, A. I., \& Mulchaey, J. S. 1998, ApJ, 496, 39

Zentner, A. R., Berlind, A. A., Bullock, J. S., Kravtsov, A. V., \& Wechsler, R. H. 2005, ApJ, 624, 505 